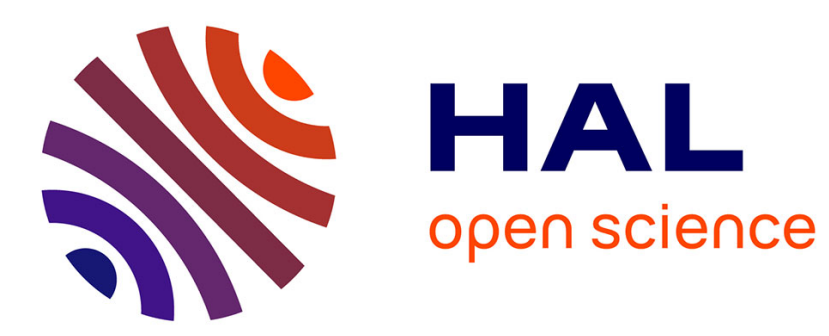

\title{
Classical Homomorphisms Between Refined Neutrosophic Rings and Neutrosophic Rings
}

\author{
Mohammad Abobala
}

\section{To cite this version:}

Mohammad Abobala. Classical Homomorphisms Between Refined Neutrosophic Rings and Neutrosophic Rings. International Journal of Neutrosophic Science, 2020, 5, pp.72 - 75 . 10.5281/zenodo.3819018 . hal-02876519

\section{HAL Id: hal-02876519 https://hal.science/hal-02876519}

Submitted on 10 Aug 2020

HAL is a multi-disciplinary open access archive for the deposit and dissemination of scientific research documents, whether they are published or not. The documents may come from teaching and research institutions in France or abroad, or from public or private research centers.
L'archive ouverte pluridisciplinaire HAL, est destinée au dépôt et à la diffusion de documents scientifiques de niveau recherche, publiés ou non, émanant des établissements d'enseignement et de recherche français ou étrangers, des laboratoires publics ou privés. 


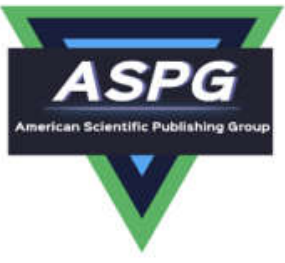

\title{
Classical Homomorphisms Between Refined Neutrosophic Rings and Neutrosophic Rings
}

\author{
Mohammad Abobala \\ Faculty of Science, Tishreen University, Lattakia, Syria. \\ E-mail: mohammadabobala777@gmail.com
}

\begin{abstract}
The aim of this paper is to study homomorphisms between refined neutrosophic rings and neutrosophic rings. We prove that every neutrosophic ring $R(I)$ is a homomorphic image of the refined neutrosophic ring $R\left(I_{1}, I_{2}\right)$. Furthermore, we prove the following interesting result:
\end{abstract}

Theorem. Let $R$ be a ring with $\operatorname{Char}(R)=2$, then $R\left(I_{1}, I_{2}\right) / K \cong R(I)$, where $K$ is a subring of $R\left(I_{1}, I_{2}\right)$ with the property $K \cong R$.

Keywords: Neutrosophic Ring, Refined Neutrosophic Ring, Homomorphic Image.

\section{Introduction}

Neutrosophy as a generalization of classical logic introduced by Smarandache plays an important role in algebraic studies, many neutrosophic algebraic structures were defined and studied such as neutrosophic groups, and neutrosophic fields. See[4, 5, 6, 8]. Neutrosophic ring was defined in [8] as a new kind of rings. It has been studied carefully in [4, 5], many related concepts such as neutrosophic subring, neutrosophic ideal, and neutrosophic homomorphism were introduced and checked. Also, concepts of refined neutrosophic ring, refined neutrosophic subring, refined neutrosophic ideal were defined and studied in [2, 3]. Concepts such as $A H$-ideal, $A H S$-ideal, and $A H S$-homomorphisms are introduced in [1]. In [3], Adeleke, Agboola and Smarandache proved the following result:

Let $Z_{n}\left(I_{1}, I_{2}\right)$ be a refined neutrosophic ring with addition and multiplication modulo $\mathrm{n}$, then $O\left(Z_{n}\left(I_{1}, I_{2}\right)\right)=n^{3}$.

In general it is easy to check that the order of finite refined neutrosophic ring $R\left(I_{1}, I_{2}\right)=n^{3}$, where $\mathrm{n}$ is the order of the finite ring $R$. The previous result motivates us to check if there is a classical ring homomorphism between $R\left(I_{1}, I_{2}\right), R(I)$ and to investigate the kernel of a homomorphism under the assumption that if there is a classical homomorphism between $R\left(I_{1}, I_{2}\right), R(I)$. Since neutrosophic ring $R(I)$ and refined neutrosophic ring $R\left(I_{1}, I_{2}\right)$ are rings by classical meaning, we can use concepts such as classical ring homomorphism/isomorphism to obtain some properties of these rings.

All homomorphisms and isomorphisms through this paper are considered by classical meaning not neutrosophical meaning defined in $[2,3,5]$. 


\section{Preliminaries}

In this section we recall some basic notions and results regarding to neutrosophic rings.

Definition 2.1.([5]) Let $(R,+, \times)$ be a ring. $R(I)=\{a+b I: a, b \in R\}$ is called the neutrosophic ring, where $I$ is a neutrosophic element with condition $I^{2}=I$.

Remark 2.2.([2]) The element $I$ can be split into two indeterminacies $I_{1}, I_{2}$ with conditions: $\mathrm{I}_{1}^{2}=\mathrm{I}_{1}, I_{2}{ }^{2}=I_{2}, I_{1} I_{2}=I_{2} I_{1}=I_{1}$.

Definition 2.3.([2]) If $X$ is a set then $X\left(I_{1}, I_{2}\right)=\left\{\left(a, b I_{1}, c I_{2}\right) ; a, b, c \in X\right\}$ is called the refined neutrosophic set generated by $X, I_{1}, I_{2}$.

Definition 2.4.([2]) Let $(R,+, \times)$ be a ring. $\left(R\left(I_{1}, I_{2}\right),+, \times\right)$ is called a refined neutrosophic ring generated by $R, I_{1}, I_{2}$.

Theorem 2.5.([2]) Let $\left(R\left(I_{1}, I_{2}\right),+, \times\right)$ be a refined neutrosophic ring. Then it is a ring.

Definition 2.6.([1]) Let $R$ be a ring and $R(I)$ be the corresponding neutrosophic ring and

$P=P_{0}+P_{1} I=\left\{a_{0}+a_{1} I ; a_{0} \in P_{0}, a_{1} \in P_{1}\right\}$, where $P_{0}, P_{1}$ are two subsets of $R$. Then $P$ is an

a) $A H$-ideal if $P_{0}, P_{1}$ are ideals in the ring $R$,

b) $A H S$-ideal if $P_{0}=P_{1}$.

3. Main discussion

Theorem 3.1: Let $(R,+, \times)$ be a ring and $R(I), R\left(I_{1}, I_{2}\right)$ the corresponding neutrosophic ring and refined neutrosophic ring respectively. Then

a) There is a ring homomorphism $f: R\left(I_{1}, I_{2}\right) \rightarrow R(I)$ defined by $f\left(a, b I_{1}, c I_{2}\right)=a+(b+c) I$.

b) The additive group $(\operatorname{Ker}(f),+)$ is isomorphic to the additive group $(R,+)$.

Proof.(a) Let $a, b, c \in R$. Define $f: R\left(I_{1}, I_{2}\right) \rightarrow R(I)$ by $f\left(a, b I_{1}, c I_{2}\right)=a+(b+c) I$. Then $f$ is well defined, since if $\left(a, b I_{1}, c I_{2}\right)=\left(x, y I_{1}, z I_{2}\right)$, then $a=x, b=y, c=z$. Thus, $a+(b+c) I=x+(y+z) I$.

Also, $f$ is a ring homomorphism. For this, suppose that $m=\left(a, b I_{1}, c I_{2}\right), n=\left(x, y I_{1}, z I_{2}\right) \in R\left(I_{1}, I_{2}\right)$, we have:

$m+n=\left(a+x,(b+y) I_{1},(c+z) I_{2}\right)$, and

$m \times n=\left(a \times x,(a \times y+b \times y+b \times z+b \times x+c \times y) I_{1},(a \times z+c \times x+c \times z) I_{2}\right)$.

So, $f(m+n)=[a+x]+[b+y+c+z] I=f(m)+f(n)$ and

$f(m \times n)=[a \times x]+[a \times y+b \times y+b \times z+b \times x+c \times y+a \times z+c \times x+c \times z] I=$

$[a+(b+c) I] \times[x+(y+z) I]=f(m) \times f(n)$.

It is clear that $f$ is a surjective map.

(b) By the definition $\operatorname{Ker}(f)=\left\{\left(a, b I_{1}, c I_{2}\right) \in R\left(I_{1}, I_{2}\right): a+(b+c) I=0=0+0 I\right\}$. Hence $a=0, b+c=0$, this means $b=-c$. Thus, $\operatorname{Ker}(f)=\left\{\left(0, b I_{1},-b I_{2}\right): b \in R\right\}$. 
It is easy to check that $\varphi: \operatorname{Ker}(f) \rightarrow R$ defined by $\varphi\left(0, b I_{1},-b I_{2}\right)=b$ is a group isomorphism between $(\operatorname{Ker}(f),+)$ and $(R,+)$.

As a simple result from the Lagrange theorem we have:

If $R$ is finite ring, then $\frac{O\left(R\left(I_{1}, I_{2}\right)\right)}{O(R)}=o(R(I))$.

Theorem 3.2. Let $R$ be a ring, where $\operatorname{Char}(R)=2$. Thenthere is a subring of $R\left(I_{1}, I_{1}\right)$ say $K$ with the property $K \cong$ $R$ and $\mathrm{R}\left(I_{1}, I_{2}\right) / K \cong R(I)$.

Proof. Applying Isomorphism Theorem, we get $\mathrm{R}\left(I_{1}, I_{2}\right) / \operatorname{Ker} f \cong R(I)$, and $\varphi$ (defined in Theorem 3.1) is a group isomorphism between $(\operatorname{Ker}(f),+)$ and $(R,+)$. Now, we prove that $\varphi$ is a ring isomorphism under the condition $\operatorname{Char}(R)=2$.

Suppose that $m=\left(0, b I_{1},-b I_{2}\right)$ and $n=\left(0, c I_{1},-c I_{2}\right)$ are two arbitrary elements in $\operatorname{Ker}(f)$, we have:

$$
m \times n=\left(0,(b \times c-b \times c-b \times c) I_{1},(b \times c) I_{2}\right)=\left(0,[-b \times c] I_{1},[b \times c] I_{2}\right) .
$$

So, $f(m \times n)=-b \times c=b \times c=f(m) \times f(n)$.

$[-\mathrm{b} \times \mathrm{c}=\mathrm{b} \times \mathrm{c}$, since $\operatorname{Char}(R)=2]$.

By the previous aspect we find that $K=(\operatorname{Ker}(f),+, \times) \cong(R,+, \times)$. Thus, $\mathrm{R}\left(I_{1}, I_{2}\right) / K \cong R(I)$.

Theorem 3.3. Let $\mathrm{R}$ be a ring. Then there is a subring of $R(I)$ say $K$ with the property $K \cong R$ and $R(I) / K \cong R$.

Proof. Define $f: R(I) \rightarrow R$ by $f(a+b I)=a$. It is easy to check that $f$ is a surjective ring homomorphism. Thus, $R(I) / \operatorname{Ker}(f) \cong R$.

Also, $\operatorname{Ker}(f)=\{a+b I \in R(I): a=0\}=\{b I: b \in R\}=R I$, since $K=R I$ is a subring of $R(I)$ and the map $\varphi: K \rightarrow R$ defined by $\varphi(b I)=b$ is a ring isomorphism, we get the proof.

Example 3.4. Let $R=Z_{2}$. Then $R$ is a ring with respect to addition and multiplication modulo2, we have $\operatorname{Char}(R)=2$.

$\mathrm{R}\left(I_{1}, I_{2}\right)=\left\{\left(a, b I_{1}, c I_{2}\right): a, b, c \in Z_{2}\right\}$ is the corresponding refined neutrosophic ring.

$R(I)=\left\{a+b I: a, b \in Z_{2}\right\}$ is the corresponding neutrosophic ring.

Let $f$ be the ring homomorphism defined in Theorem 3.1, we have:

$\operatorname{Ker}(f)=\left\{\left(0, b I_{1},-b I_{2}\right): b \in Z_{2}\right\}=\left\{(0,0,0),\left(0, I_{1}, I_{2}\right)\right\} \cong R$ and

$\mathrm{R}\left(I_{1}, I_{2}\right) / K=\left\{\mathrm{K},(1,0,0)+\mathrm{K},\left(0, \mathrm{I}_{1}, 0\right)+\mathrm{K},\left(1,0, \mathrm{I}_{2}\right)+\mathrm{K}\right\} \cong R(I)$.

So, $O\left(R\left(I_{1}, I_{2}\right)\right)=8, O(R(I))=4, O(R)=2$. Therefore, $O\left(\mathrm{R}\left(I_{1}, I_{2}\right)\right) / O(R)=O(R(I))=4$.

\section{Conclusion}

In this paper we have studied the relationship between refined neutrosophic rings and neutrosophic rings by using classical methods in Ring Theory. In particular have shown: 
a) Every neutrosophic ring $R(I)$ is a homomorphic image of refined neutrosophic ring $R\left(I_{1}, I_{2}\right)$.

b) If $\operatorname{Char}(R)=2$, then there is a subring of $R\left(I_{1}, I_{2}\right)$ say $K$ with property $K \cong R$ andR $\left(I_{1}, I_{2}\right) / K \cong R(I)$.

Funding: This research received no external funding.

Conflicts of Interest: The authors declare no conflict of interest.

\section{References}

[1] M. Abobala, On Some Special Substructures of Neutrosophic Rings and Their Properties, International Journal of Neutrosophic Science, vol. 4, pp. 72-81, 2020.

[2] E.O. Adeleke, A.A.A.Agboola and F. Smarandache, Refined Neutrosophic Rings I, International Journal of Neutrosophic Science, vol. 2, pp. 77-81, 2020.

[3] E.O. Adeleke, A.A.A. Agboola and F. Smarandache, Refined Neutrosophic Rings II, International Journal of Neutrosophic Science, vol. 2, pp. 89-94, 2020.

[4] A.A.A. Agboola, E.O.Adeleke and S.A. Akinlye,Neutrosophic Rings II,International J.Mathcombin, vol. 2, pp. 1-8, 2012.

[5] A.A.A. Agboola, A.D.Akinola and O.Y. Oyebola, NeutrosophicRings I, International J.Mathcombin, vol. 4,pp. 1-14, 2011.

[6] T. Chalabathiand K. Kumar, Neutrosophic Units of Neutrosophic Rings and Fields, Neutrosophic Sets and Systems, vol. 21, pp. 5-9, 2018.

[7] M. Haushi, Algebraic Structures 2, Tishreen University Press, pp. 132-190,2004.

[8] V.W.B. Kandasamy and F. Smarandache, Some Neutrosophic Algebraic Structures and Neutrosophic N-Algebraic Structures, Hexis, Phonex, Arizona 2006. 